\title{
Bilateral Erosive Hand Osteoarthritis in a 46-Year-Old Woman
}

\author{
Danjem $\mathrm{SM}^{1^{*}}$, Salaam $\mathrm{AJ}^{1}$ and Ibinaiye $\mathrm{PO}^{2}$ \\ ${ }^{1}$ Radiology Department, University of Jos, Jos University Teaching Hospital, Jos, Nigeria \\ ${ }^{2}$ Radiology Department, Ahmadu Bello University, Ahmadu Bello University Teaching Hospital, Zaria, Nigeria
}

*Corresponding author: Danjem SM, Radiology Department, University of Jos, Jos University Teaching Hospital, Jos, Nigeria, Tel: 08061206009, E-mail: samueljiblik@gmail.com; sdanjem@yahoo.com

Citation: Danjem SM, Salaam AJ, Ibinaiye PO (2018) Bilateral Erosive Hand Osteoarthritis in a 46-Year-Old Woman. Saj Case Rep 5: 405

Article history: Received: 28 June 2018, Accepted: 14 September 2018, Published: 17 September 2018

\begin{abstract}
Purpose: To describe classical radiographic appearances of erosive arthritis of the hands in an adult woman. Case Report: A 46-year-old woman who presented in the Clinic at a private hospital, with pain, swelling, morning stiffness, deformity of the fingers and occasional difficulty in using both hands. She had used various nonsteroidal anti-inflammatory drugs (NSAIDs) including Ibuprofen which was prescribed in a peripheral hospital, but her complaints worsened with no relief. Plain radiograph of the hands showed typical features of Erosive Osteoarthritis (EOA) in distal interphalangeal (DIP) and proximal interphalangeal (PIP) joints such as joint space narrowing, subchondral sclerosis, marginal osteophytes and erosions in the central portion of the joint giving the "gull-wing" and "saw tooth" appearances. The patient was placed on hydroxychloroquine, colchicines, piroxicam and prednisolone. Conclusion: A case of a 46-yearold woman with bilateral erosive hand osteoarthritis was reported. The rarity of the condition especially in our environment and the classical radiographic features prompted the report of this case.
\end{abstract}

Keywords: Erosive Osteoarthritis; Hands; Radiographic Features

\section{Introduction}

Erosive osteoarthritis (EOA) is an uncommon subset of osteoarthritis (OA) characterized by both inflammatory and degenerative phenomena of the distal interphalangeal (DIP) and proximal interphalangeal (PIP) joints of the hand. It less frequently affects the first carpometacarpal joint [1]. EOA is also known as an inflammatory form of OA. The exact prevalence of EOA is unknown. Kwok, et al. documented a prevalence of $2.8 \%$ EOA in the general population. The common symptoms include hand pain and disability to use the hand [2]. The detection of synovial membrane inflammation and tenosynovitis in most patients with EOA is consistent with the term of inflammatory OA Radiological imaging is the standard method to study the progression and the successive pathological changes of erosive osteoarthritis. The classical radiographic appearance prompted the report of this case $[3,4]$.

\section{Case Report}

A 46-year-old woman who presented in the Clinic at a private hospital, with pain, morning stiffness, deformity of the fingers and occasional difficulty in using both hands. She had used various nonsteroidal anti-inflammatory drugs (NSAIDs) including Ibuprofen which was prescribed in a peripheral hospital, but her complaints worsened with no relief. No positive family history of such condition. On examination, the patient showed changes of DIP and PIP joints characterized by tenderness and decreased range of motion. The metacarpophalangeal (MCP) joints, wrist and foot joints were not affected. The skin and nails appeared normal.

Plain radiograph of the hands showed typical features of EOA in DIP and PIP joints such as joint space narrowing, subchondral sclerosis, marginal osteophytes and erosions in the central portion of the joint giving the "gull-wing" and "saw tooth" appearances. Angular deformity of the DIP and PIP joints of the second and third fingers bilaterally was noted. Associated overlying soft tissue swelling was also noted bilaterally (Figure 1).

Laboratory findings showed a raised erythrocyte sedimentation rate (ESR) of $34 \mathrm{~mm} / \mathrm{h}$ (normal <20) and C-reactive protein (CRP) of $13 \mathrm{mg} / \mathrm{l}$ (normal $<5 \mathrm{mg} / \mathrm{l}$ ). The patient was negative for rheumatoid factor (RF). Routine biochemistry was normal. Using the radiological findings, as well as the laboratory results a diagnosis of EOA was made and the patient was referred back 
to the MOPD for further management. The patient was placed on medication consisting of hydroxychloroquine (200mg/day), colchicines (1.5mg/day), piroxicam ( $10 \mathrm{mg} /$ day), but this treatment did not alleviate the patient's pain, hence, prednisolone (10 mg/ day) was added, and the hydroxychloroquine dose was raised to $400 \mathrm{mg} /$ day. Four weeks after the initiation of prednisolone, there was considerable improvement in the pain and morning stiffness. Additionally, ESR and CRP decreased to normal levels (15 mm/h and $3.01 \mathrm{mg} / \mathrm{l}$ respectively). On follow up at the clinic, the patient's symptoms had remarkably improved.

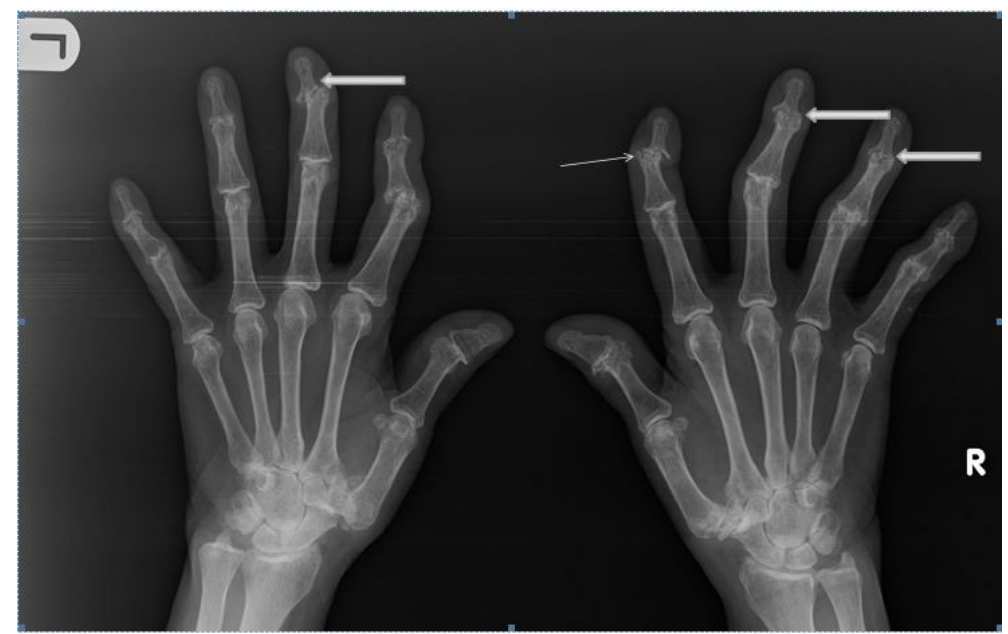

Figure 1: Conventional radiograph postero-anterior view of both hands showing joint space narrowing of all the DIP joints bilaterally and subluxation of the third left DIP, fourth left PIP joints and second, third right DIP joints, third, fourth PIP joints. Remarkably, MCP joints and wrists are spared. Note "gull-wing" appearances (thick arrows) in the distal plate of third left and second, third fourth left DIP joints; "saw-tooth" (thin arrow) appearance in the second right DIP joint. Marginal osteophytes are also noted. Carpal joint space narrowing is also shown. Mild overlying soft tissue swelling is also demonstrated over the PIP and DIP joints

\section{Discussion}

Erosive osteoarthritis is a progressive disorder with a typically bilateral and symmetrical distribution in the interphalangeal joints and less frequently the first carpometacarpal joint of the hand or the foot joints. Radiologically, central erosions which typically show the classic "gull-wing" or "saw-tooth" deformity characterize this disorder [1,3]. Kwok, et al. estimated the prevalence of EOA to be $2.8 \%$ in the general population and $10.2 \%$ in individuals with symptomatic hand osteoarthritis (HOA) [2]. Recently Haugen, et al. reported apparently higher prevalence estimates of erosive OA (9.9\% for women and 3.3\% for men ages 40-84 years) [5]. Erosive osteoarthritis, like more severe hand osteoarthritis, targets the DIP joints more frequently than the PIP joints [6,7]. The exact etiology of EOA is not known, but several studies were carried out to find the possible etiological factors of EOA. Marion, et al. suggested that underlying systemic processes are involved in the evolution of EOA based on the fact that inflammatory signs are more frequent in EOA than in non-EOA. The exact mechanism is not yet known, BMI was found to be increased in individuals with erosive osteoarthritis compared with non-erosive OA suggesting that patients with EOA may have a selected metabolic risk factor, notably dyslipidemia [6,9]. Marshall, et al. documented that the frequency and pattern of joint involvement in erosive osteoarthritis is similar to that seen in moderate-severe and severe non-erosive radiographic osteoarthritis, implies that EOA complicates non-erosive hand osteoarthritis. This means that EOA is a more severe form of hand osteoarthritis rather than a distinct entity [6]. Other risk factors, such as heredity and female predominance have also been reported for erosive osteoarthritis [11]. No known cause or risk factor was noted in the current case.

Patients with EOA clinically present with pain, swelling, redness, warmth and limited function of the interphalangeal joints (IPJs) of the hands [11]. These features were also reported by Kwok, et al. reported EOA involving the first carpometarcarpal joint (CMCJs) in $2.2 \%$ of patients with hand pain which was not accompanied by erosions in IPJs [2].

Although the diagnosis of EOA rests upon careful radiographic reading, analysis of clinical and laboratory findings are the keys to differentiating this disease from other more common interphalangeal arthropathies such as rheumatoid arthritis (RA), psoriatic arthritis (PsA), chronic tophaceous gout, chronic renal diseases and endocrine diseases. Laboratory findings, such as ESR, CRP, ANA, RF are usually negative in patient with EOA, but a slight increase of ESR and CRP may occur. Similar slight increase in ESR and CRP was documented in the present case [12]. In the present case, the distribution of articular lesions ruled out the diagnosis of RA as DIP joint involvement occurs less commonly. The anti-cyclic citrullinated peptide (anti-CCP) antibodies and RF can be considered a further useful test to help discriminate between EOA and RA [13]. The negative RF test in the present case, made the diagnosis of RA unlikely and argued for the diagnosis of EOA. Additionally, the presence of characteristic radiological findings including osteophytes, subchondral sclerosis and central erosions which gave "gull-wing" and "saw tooth" appearances was consistent with EOA. Central erosions, subchondral sclerosis and osteophytes are not typically seen in RA. The typical radiographic findings of RA include periarticular osteopenia and marginal erosions [3]. 
Interphalangeal joint involvement is a common feature of psoriatic arthritis (PsA), as well as an asymmetrical pattern of articular involvement, psoriatic skin, nail abnormalities, and a family history of psoriasis. Additionally, similar to RA, PsA causes marginal erosions giving a "mouse ear" appearance in the interphalangeal joints. Furthermore, in PsA the erosions occur not only in the DIP and PIP joints, but in MCP and wrist joints as well [3,13]. Notably, all these were negative in the current case. Severe interphalangeal joint destruction with relative sparing of the MCP joints is a typical feature of EOA. An important clue in distinguishing the advanced stages of RA and PsA from EOA is the absence of bone erosion in the MCP joints in the latter. Although degenerative changes occasionally occur in the MCP joints in EOA, they are not characterized by surface erosions. However, the erosions in these joints in RA and PsA are often conspicuous [3,13]. In consonance with EOA, the MCP joints were preserved in the present case.

The use of ultrasound was employed by Marion et al. and reported more inflammatory signs such as increased Power Doppler signal (PDS) and synovial fluid in the IPJs of patient with EOA than the IPJs of patients with non-EOA [8]. Ultrasound was not done in the current case because of lack of high frequency transducer which was employed by Mario, et al. to produce images with high resolution [8].

Treatment of EOA is aimed at controlling the inflammatory processes and alleviates pain. There are currently no guidelines on the best therapeutic approach for EOA. The available treatments are limited and include analgesics, NSAIDs and intra-articular injection of corticosteroids [1].

\section{Conclusion}

A 46-year-old woman with bilateral erosive hand osteoarthritis who presented with hand pain, deformity and inability to use the hands. The classical plain radiographic features of gull-wing saw tooth appearance with angular deformity of the DIP and PIP joints of the second and third fingers bilaterally. The ESR was mildly raised; the C-reactive protein was normaland the patient was treated with colchicine, piroxicam, hydroxychloroquine and prednisolone with significant relief of symptoms.

\section{References}

1. Punzi L, Ramonda R, Sfriso P (2004) Erosive osteoarthritis. Best Pract. Res Clin Rheumatol 18: 739-58.

2. Kwok WY, Kloppenburg M, Rosendaal FR, Van Meurs JB, Hofman M, Bierma-Zeinstra SMA. Erosive hand osteoarthritis: its prevalence and clinical impact in the general population and symptomatic hand osteoarthritis. Ann Rheum Dis 70: 1238-42

3. Martel W, Stuck KJ, Dworin AM, Hylland RG (1980) Erosive osteoarthritis and psoriatic arthritis: A radiologic comparison in the hand, wrist, and foot. Am J Roentgenol 134: 125-35.

4. Keen HI, Wakefield RJ, Grainger AJ, Hensor EM, Emery P, et al. (2008) Can ultrasonography improve on radiographic assessment in osteoarthritis of the hands? A comparison between radiographic and ultrasonographic detected pathology. Ann Rheum Dis 67: 1116-20.

5. Haugen IK, Englund M, Aliabadi P, Niu J, Clancy M, et al. (2011) Prevalence, incidence and progression of hand osteoarthritis in the general population: the Framingham Osteoarthritis Study. Ann Rheum Dis 70: 1581-6.

6. Marshall M, Nicholls E, Kwok WY, Peat G, Kloppenburg M, et al. (2013) Erosive osteoarthritis: a more severe form of radiographic hand osteoarthritis rather than a distinct entity? Ann. Rheum Dis 1: 1-6.

7. Addimanda O, Mancarella L, Dolzani P Punzi L, Fioravanti A et al. (2012) Clinical and radiographic distribution of structural damage in erosive and nonerosive hand osteoarthritis. Arthritis Care Res 64: 1046-53.

8. Marion CK, Kwok WY, Reijnierse M, Tom WJ, Kloppenburg M (2013) In erosive hand osteoarthritis more inflammatory signs on ultrasound are found than in the rest of hand osteoarthritis. Ann Rheum Dis 72: 930-4.

9. Kwok WY, Kloppenburg M, Rosendaal FR, van Meurs JB, Hofman A, et al. (2011) Erosive hand osteoarthritis: its prevalence and clinical impact in the general population and symptomatic hand osteoarthritis. Ann Rheum Dis 70: 1238-42.

10. Kloppenburg M, Kwok WY (2011) Hand osteoarthritis-a heterogeneous disorder. Nat Rev Rheumatol 8: 22-31.

11. Punzi L, Frigato M, Frallonardo P (2012) Inflammatory osteoarthritis of the hand. Best Pract. Res Clin Rheum 24: 301-12.

12. Punzi L, Ramonda R, Oliviero F, Sfriso P, Mussap M, et al. (2005) Value of C reactive protein in the assessment of erosive osteoarthritis of the hand. Ann Rheum Dis 64: 955-7.

13. Morozzi G, Bellisai F, Fioravanti A, Galeazzi M (2005) Absence of anti-cyclic citrullinated peptide antibodies in erosive osteoarthritis: further serological evidence of the disease as a subset of osteoarthritis. Ann Rheum Dis 64: 1095-6. 